\title{
凤鲚繁殖群体的生物学特性 及因数关系的研究
}
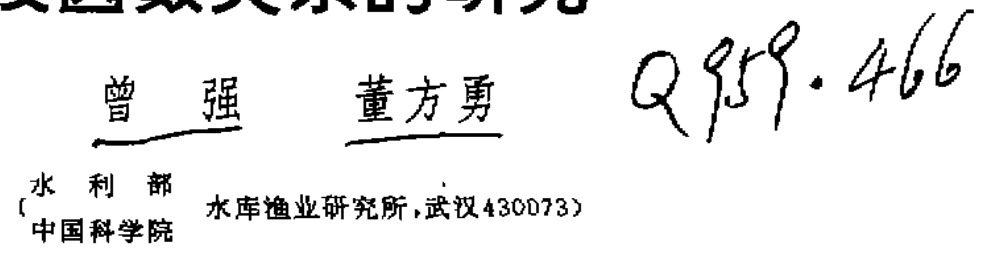

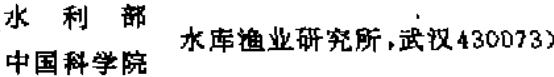

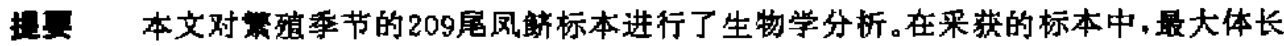
$182 \mathrm{~mm}$, 体重 $23.4 \mathrm{~g}$ ：最小体长 $94 \mathrm{~mm}$ ，体重 $3.5 \mathrm{~g}$ 。肥满度 $(k)$ 平均为 $3.15 \times 10^{-4}$ 。其体长与体重的

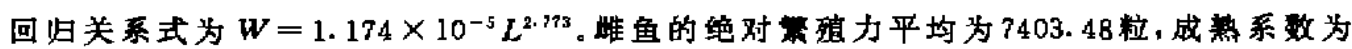

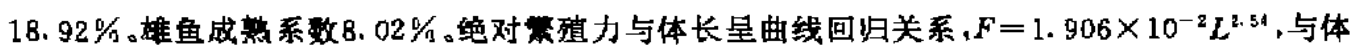
重旺直线回归关系, $F=688.13 W-2119.68$ 。

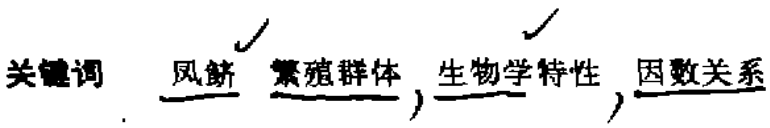

凤鲛 [Coilia mystus (Linnaeus)]属鲱形目 (Clupei-formes) 鲱科 (Clupeidae) 䲞属 (Coilia), 是一种小型鱼类, 我国沿海均产有 ${ }^{[8]}$, 在长江口一带主要分布于崇明至江阴。凤魰 届河口性洄游鱼类, 平时栖息于沿海, 每年春季大量鱼群洄游到长江口咸淡水区域产卵,但 不上湖至纯淡水区。当年出生的幼鱼在崇明一带深水处生长, 以后回到海中。凤魰是长江口 地区的主要经济鱼类之一, 在生殖季节集群繁殖而形成渔湃, 其肉味鲜美, 不论鲜食或制成 蠸头, 均为人们所喜爱。

凤鲛的生物学前人曾做过一些研究 ${ }^{[3.7 .9 .10]}$, 但慗殖时期的生物学因数关系的报道较帘 见。本文对风铈繁殖群体的生物学特性及因数关系进生了初步研究。

\section{一、材 料 和 方 法}

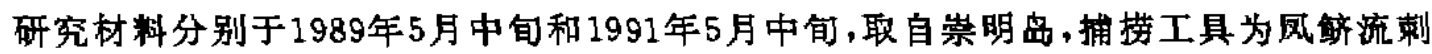
网。两饮共收集风魰标本209尾, 其中堆鱼180尾、雄鱼29尾。分别测量了体长、体重、空壳体 重、性腺重、绝对坏卵量、卵径大小等参数。对风鲛的体长与体重、绝对䇣殖力与体长、体重的 一些主要生物学参数进行了相关因子的数据分析。将所测数据在计算机上进行演算, 应用程 库为 Lotus 1-2-3。体长与体重相关关系用 $W=a L^{b}$ 公式进行计算分析; 体长与绝对繁殖力相 关关系用 $F=a L^{b}$ 、体重与绝对繁殖力相关关系用 $F=a+b W$ 公式进行计算分析。利用公式

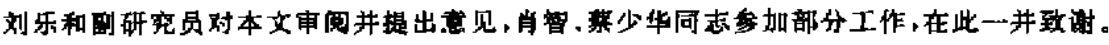

本文于1992年3月6日收到,6月1日改回。 


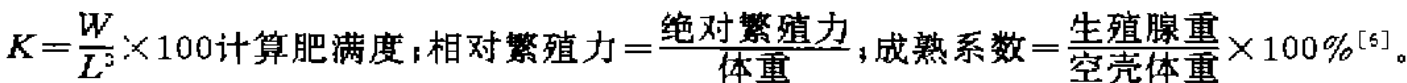

\section{二、结 果}

\section{1. 体长、体亚参数}

采集到的风鲛标本,从外部形态观察有较大差异,通常是雌鱼个体大、雄鱼个体小。在㢣

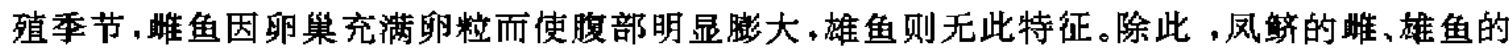
外部结构无较大差异。鱼体测量结果见表1。

表1 凤鲛体长、体重参数

Tab, 1 Body length and body weight of Coilia mystus

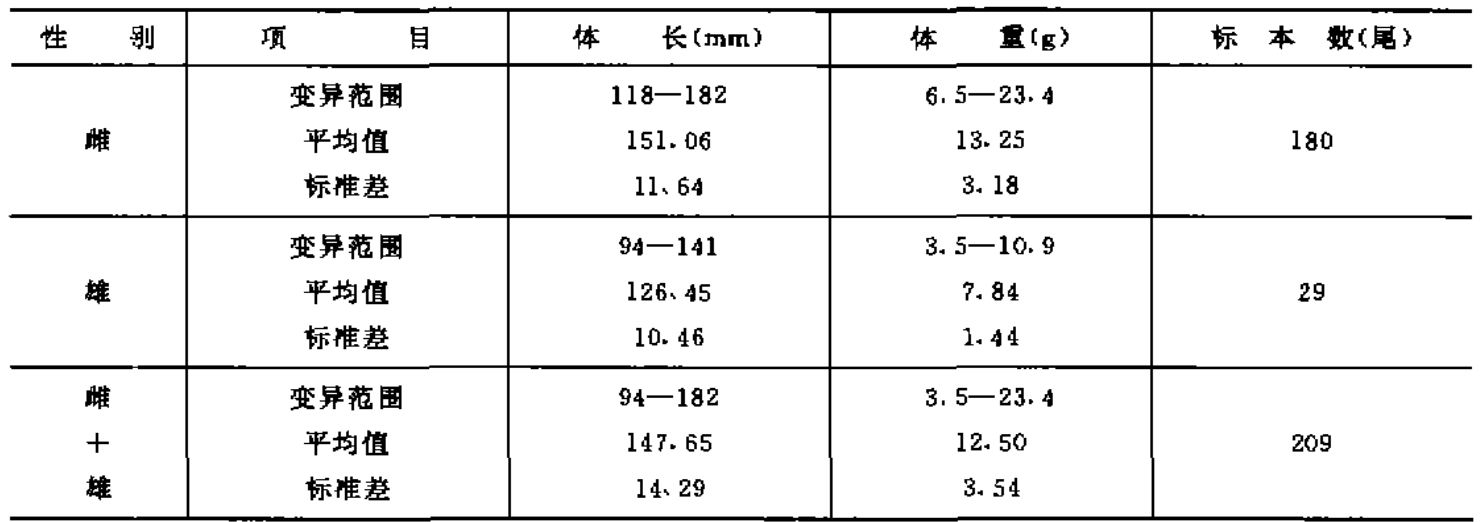

表2 凤餢各体长组出现的频数

Tab. 2 Frequency of Coilia myst us in various body-length groups

\begin{tabular}{|c|c|c|c|c|c|c|c|}
\hline \multirow{2}{*}{ 体 $\begin{array}{c}\text { 长 } \\
(\mathrm{mm})\end{array}$} & \multirow[t]{2}{*}{ 组 } & \multicolumn{3}{|c|}{ 出 现 频 数 } & \multicolumn{3}{|c|}{ 出现 韧 } \\
\hline & & 隺 & 雄 & 总和 & 占雌鱼 & 占婎鱼 & 占总数 \\
\hline $90-99$ & & 0 & 2 & 2 & 0 & 6.90 & 0.96 \\
\hline $100-109$ & & 0 & 0 & 0 & 0 & 0 & 0 \\
\hline $110--119$ & & 1 & 1 & $2^{-}$ & 0.56 & 3.45 & 0.96 \\
\hline $120-129$ & & 2 & 16 & $1 B$ & 1.11 & 55.17 & 8. 61 \\
\hline $130--139$ & & 21 & $?$ & $2 B$ & 11.67 & 24,14 & 13.40 \\
\hline $140-149$ & & 64 & 3 & 67 & 35.56 & 10.35 & 32.06 \\
\hline $150-159$ & & 52 & 0 & 52 & 28.89 & 0 & 24.88 \\
\hline $160-169$ & & 25 & 0 & 25 & 13.89 & 0 & 11.96 \\
\hline $170-179$ & & 11 & 0 & 11 & 6.11 & 0 & 5.26 \\
\hline $180-$ & & 4 & 0 & 4 & 2.22 & 0 & 1.91 \\
\hline
\end{tabular}

从表2、图1中可看出风鲚崔、雄鱼体长组的分布趋势,雌鱼体长主要分布于 $140 \mathrm{~mm}$ 组和 $150 \mathrm{~mm}$ 组, 出现频数分别为 64 尾和 52 尾, 占崔鱼总数的 $35.56 \%$ 和 $28.89 \%$ 。雄鱼主要分布在 $120 \mathrm{~mm}$ 组, 出现频数为 16 尾, 占雄鱼总数的 $55.17 \%$ 。 


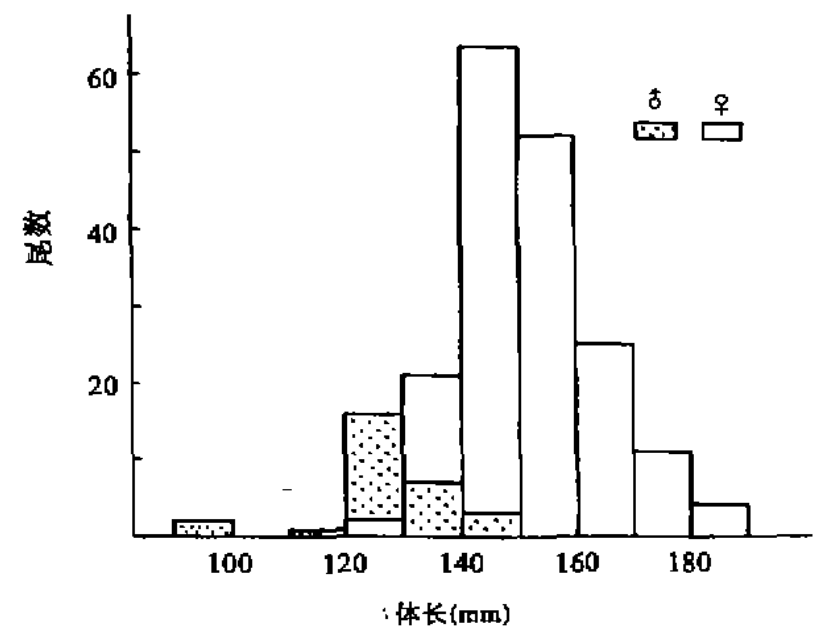

图1 各体长组风瞄崔雄出现版数

Fig. 1 Frequency of the numbers of female and male of Coilia mystus in various body-length groups

利用公式 $K=\frac{W}{L^{3}} \times 100$ 计算风鿷在繁殖季节的肥满度，其结果见表 3 。

表3 风锌的肥满度 $(K$ )

Tab. $3 \quad K$ Value of Coilia mystus

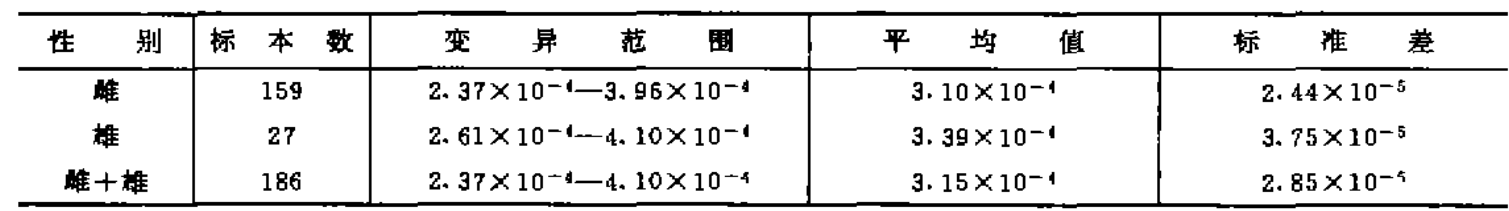

从表 3中看出, 同魰在繁殖季节肥满度 $(K)$ 变异范围在 $2.37 \times 10^{-4}-4.10 \times 10^{-4}$, 平均

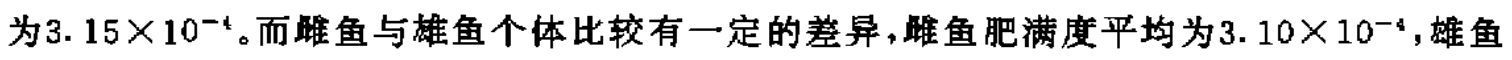
平均为 $3.39 \times 10^{-4}$, 则堆鱼的肥满度小于雄鱼。

\section{2. 体长与体而相关关柔}

鱼类的体长与体重之间存在一定的函数关系, 但种类不同的鱼类,两者之间的函数关系 亦不同。根据风鲛标本测量的数据分析, 其体长与体重相关关系适合于需函数关系 $(W=$ $\left.a L^{b}\right)$, 这与大多数鱼类的体长与体重相关关系是相同的 ${ }^{[1,4,5.6,11]}$. 利用有关测量的数据, 确定 函数关系式即 $W=a L^{b}$ 中的 $a 、 b$ 值以及相关系数 $r$,并计算出相关系的 $F$ 检验值、其结果 见表4。得出体长与体重关系的回归方程式为:

$W=1.090 \times 10^{-5} L^{2.789}$, 相关系数 $F$ 检验， $F(972.61)>F_{\text {0. } 01}(6.64)$ ，相关极显著、雄鱼 $W=1.394 \times 10^{-4} L^{2.257}$, 经 $F$ 检验， $F(131.45)>F_{0.01}(7.68)$, 相关极显著。根据计算值可绘 出雌、雄鱼体长与体重的回归曲线图（图2)。由图可见、雄、雄鱼体长与体重相关回归曲线有 一定差异,两条曲线在体长约为 $120 \mathrm{~mm}$ 处相交。体长在 $120 \mathrm{~mm}$ 左右以下时,同等体长雄鱼 
的体重大于雌鱼; 而在体长 $120 \mathrm{~mm}$ 以上时，同等体长崔鱼的体重大于雄鱼体重。这种现象随 体长的增长，差异越来越大。勧鱼与雄鱼混合的体长与体重相关关系式则为: $W=1.174 \times$ $10^{-5} L^{2773}$, 经 $F$ 检验. $F(1824.40)>F_{00}(6.64)$ ，相关极显著。根据体长与体重实测数据和 计算出的相关关系式数据、可绘出崔、雄混合的体长与体重相关回归曲线图(图3)。经分析， 唯雄混合的体长与体重相关回归曲线与略鱼的回归曲线近似。

表4 凤鯍体长与体重相关固子数

Tab. 4 The relative factors between body length or body weight of Coilia mystus

\begin{tabular}{|c|c|c|c|c|c|}
\hline 性 & 标 本 数 & 䢂 & $b$ & $r$ & $F$ \\
\hline 堆 & 180 & -4.963 & 2.789 & 0.919 & 972.61 \\
\hline 雄 & 29 & -3.856 & 2.257 & 0.911 & 131.45 \\
\hline 堆十雄 & 209 & -4.930 & 2. 773 & 0.948 & 1824.40 \\
\hline
\end{tabular}

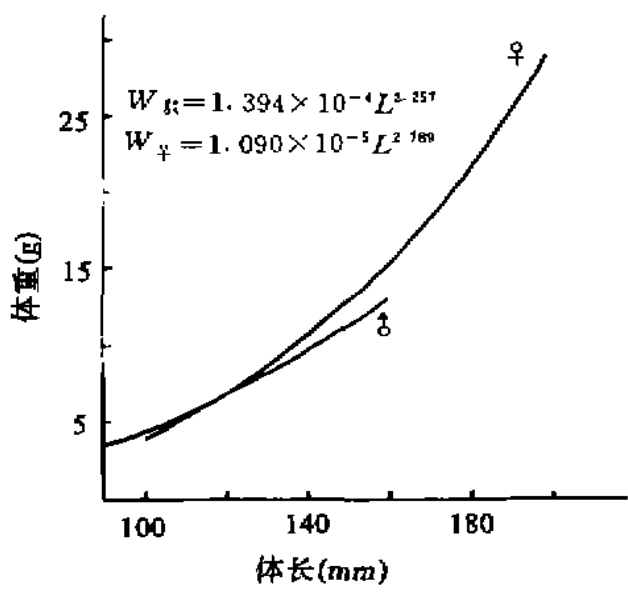

国2 凤鰙㫿、雄体长与体重相关回归曲线

Fig. 2 Regression curve for body length and body weight of female and male of Coilia mystus

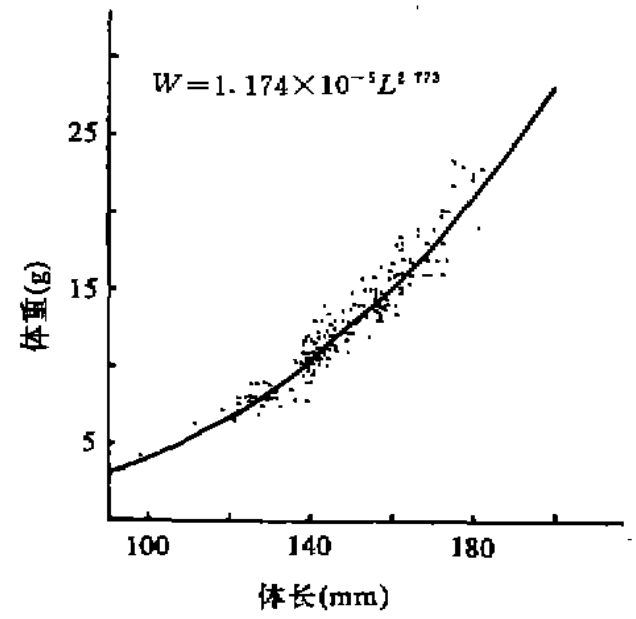

图3 凤斯醮雄混合体长与体重相关回旧曲线

Fig. 3 Regression curve for body length and body weight of medley of female and male of Coilia mystus

\section{3. 策殖}

据资料记载.凤鲚均为 1 龄个体 ${ }^{-9]}$ 。作者收集的标本经鉴定亦均为 1 龄, 未见 1 龄以上的个 体。由此推断, 凤鲛的生命周期较短, 亲鱼产卵后不久即死亡。繁殖产卵群体均为补充群体。

收集了209尾风鿷样品, 其中崔鱼为180尾, 而雄鱼仅为 29 尾, 崔雄鱼性比为6.211。经解 剖观察,样品中155尾崔鱼的卵巢和28尾雄鱼的精巢处 $N$ 期未。少数个体为 $\mathrm{V}$ 期或 V 期,已产 卵(半产或全产)或已排精,其中雌鱼25尾、雄鱼1尾。对156尾(其中，睢鱼130尾、雄鱼26尾)进 行警殖力研究,结果见表 5 。 


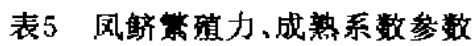

Tab. 5 The fecondity and maturity coefficient of Coilsa mystus

\begin{tabular}{|c|c|c|c|c|c|}
\hline 别 & 标本 数 & 项 & 变 异 萢 围 & 平均 值 & 标 准 考 \\
\hline 蜼 & 130 & 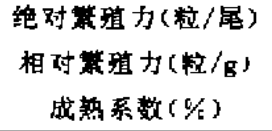 & $\begin{array}{c}1968-18304 \\
202.5-1102.7 \\
9.6-28.6\end{array}$ & $\begin{array}{r}7403.48 \\
526.52 \\
16.92\end{array}$ & $\begin{array}{r}3414.87 \\
172.72 \\
4.08\end{array}$ \\
\hline 雄 & 26 & 成热系数 (\%) & $5.0-12.5$ & B. 02 & 1.96 \\
\hline
\end{tabular}

\section{4. 等殖力与体长、体亚的相关关系}

测量了风鲛雌鱼 130 尾标本的绝对繁殖力和体长、体重数据, 并进行统计分析, 结果其绝 对熬殖力与体长的相关关系符合㫷函数关系，与体重的相关关系呈直线关系。

把测量的数据、利用计算机里的 Lotus 1-2-3程序、计算出绝对繁殖力与体长的相关关 系式 $W=a L^{b}$,与体重的相关关系式 $F=a+b W$ 中的相关因子 $a 、 b$ 和相关系系数 $r$, 以及相 关系数的 $F$ 检验值。结果见表 6 。得出绝对繁殖力与体长的相关关系式为: $F=1.906 \times 10^{-2}$ $L^{254}$, 经 $F$ 检验, $F(38.23)>F_{0.01}(6.85)$ ，相关极显著，绝对繁殖力与体重的相关关系式为 $F$ $=688.13 W-2119.68 . F$ 检验, $F(102.14)>F_{0.01}(6.85)$, 相关极显著。绝对繁殖力与体长、 体重的回归曲线见图4、5, 随着其个体体长的增长和体重的增加, 它的绝对繁殖力也相应增 如。

\section{表6 风既绝对策殖力与体长、体重相关因子參数}

Tab. 6 The relative factors between body length or body weight and absolute fecondity of Coilia mystus

\begin{tabular}{|c|c|c|c|c|c|}
\hline & 标 本 数 & $a$ & $b$ & $r$ & $F$ \\
\hline 能对第邀力一体长 & 130 & -1.72 & 2.54 & 0.480 & 38.23 \\
\hline 绝对需殖力一体重 & 130 & & 688.13 & 0.671 & 104.73 \\
\hline
\end{tabular}

\section{5. 卵径}

风鲛成熟的卵子呈黄色略带青灰色。抽取 18 尾标本测量卵径结果、卵径变化幅度为 $0.68-0.91 \mathrm{~mm}$. 平均为 $0.83 \mathrm{~mm}$, 标准差 $0.057 \mathrm{~mm}$ 。

\section{三、小结与讨论}

凤魰是一种小型经济鱼类、虽然它的个体小, 但种群量很大。在长江口渔场每年 $4-7$,月 份为其渔汛季节,产量占总渔获量的 $25 \%$ 左右,居于首位 ${ }^{[7]}$ 。

凤鲛崔雄个体大小相差较大, 倠鱼平均体长 $151.06 \mathrm{~mm}$, 平均体重 $13.25 \mathrm{~g}$, 雄鱼为 $126.45 \mathrm{~mm}$ 和 $7.84 \mathrm{~g}$, 唯鱼明显大于雄鱼。繁殖时期由于体内大量营养物质供给了卵柴发育、 此时肥满度 $K$ 值, 则是雄鱼 $\left(3.39 \times 10^{-4}\right)$ 略大于崔鱼 $\left(3.10 \times 10^{-4}\right)$ 。

风魰的寿命、前人未详细研究过。据有关文献记载 ${ }^{[9]}$, 镂片呈圆型且薄,轮纹排列不规 则, 未见年轮。作者曾对风鲚的鳞片和脊推骨进行了观察, 镂片的轮纹排列没有年轮反映,与 文献记述相同。脊椎骨上则可看出黑白交替的同心宽狭环纹,均仅有一条暗色的狭纹靠近背 


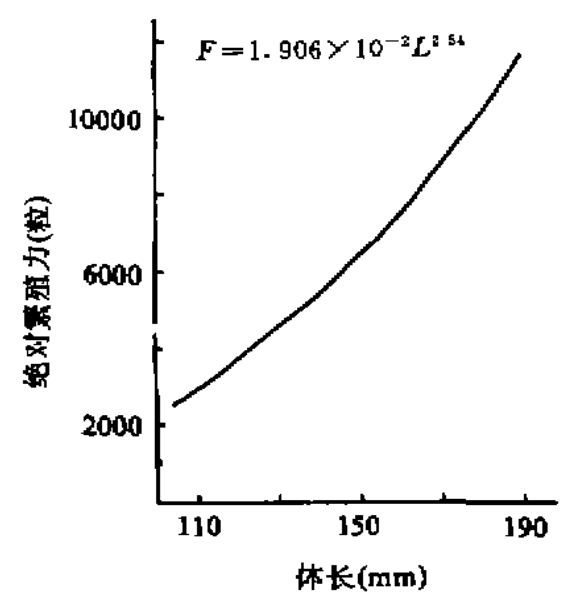

图4 风镜体长与绝对等殖力相关曲线

Fig. 4 Regression curve for body length and absolute fecondity of Coilia mystus

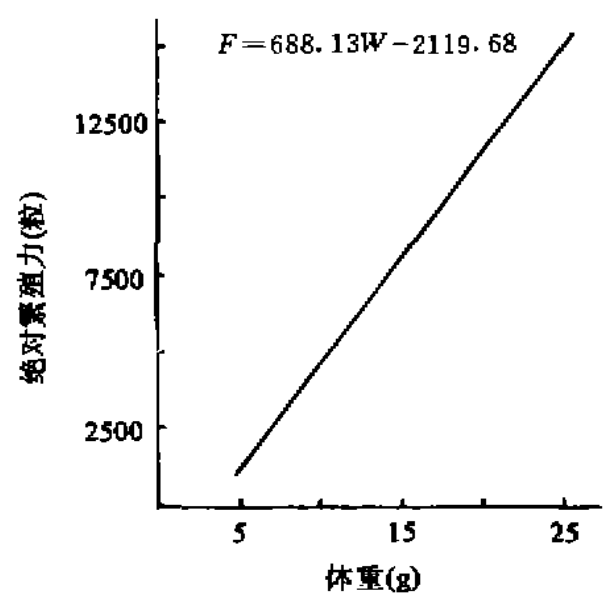

图5 风鲸体重与绝对策殖力相关直线

Fig. 5 Regression straight line for body weight and absolute fecondity of Coilia mystus

椎骨的边缘, 表明一个年层。据此认为采集的风鲛慗殖群体都是 1 齢个体。

堆雄鱼个体的性比为6.2:1, 蜼性远多于雄性, 其原因可能与捕措工具有关。流刺网网目 较大, 捕拱到的大多为崔鱼, 雄鱼只占少数。再者, 由于雄鱼个体小, 游泳能力弱,一般沿江常 上溯,故深水定置张网中捕获的主要是雄鱼个体 ${ }^{[2]}$ 。

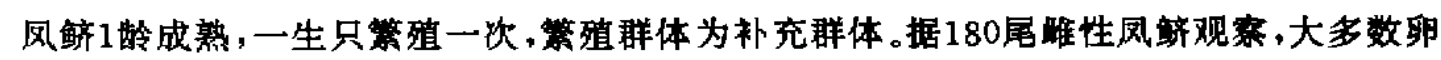
笨饱满,处 $N$ 期末, 它们的绝对繁殖力在 1968 -18304粒, 平均 7403.48粒。这与袁传密 ${ }^{[0]}$ 报道 的风鲛绝对繁殖力一般为 4-5万粒相比要偏低,而接近湖北省水生生物研究所鱼类研究 室 ${ }^{[r]}$ 报道的风鲸绝对繁殖力约在 $5000-20000$ 粒。平均成就系数 $18.92 \%$ 、平均卵径 $0.83 \mathrm{~mm}$, 这与过去文献记载较相近。

[1] 王良臣等.黄塔生物学因数关系的研究.鱼类学论文重, 1985,(4), 147-153.

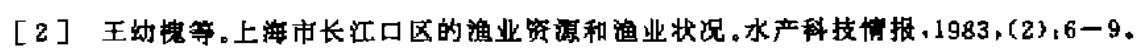

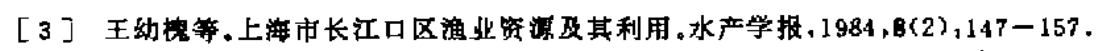

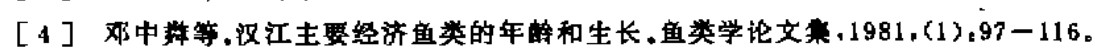

$[5]$ 华元消等.鱼种重量与长度相关公式 $\left(W=6 L^{a}\right)$ 的生物学意父及其运用。鱼类学论文蕃, $1981,(1): 125-132$.

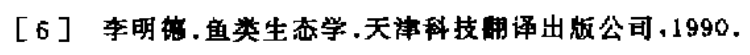

[7] 张国样等。长江口定賚张网汿业调查。水产学报, 1985,9(2): 186-198.

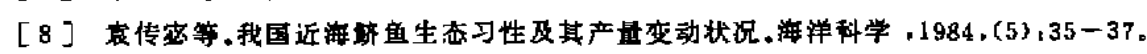

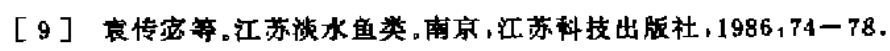

[10] 湖北省水生生物研究所鱼类研究室。长江鱼类.北京、科学出版扗.1976,25-27.

[11]解玉浩等。水丰水库的池沼公鱼生物学.水生生物学集刊, 1984, B(4), 457-467。 


\title{
STUDY ON THE BIOLOGICAL CHARACTERISTICS AND FACTOR CORRELATION OF COILIA MYSTUS PROPAGATING POPULATION
}

\author{
Zeng Qjang Dong Fangyong \\ (Institute of Reservatr Fisheries, Ministry of Water Resources and Academta Sinica, Wuhar)
}

\begin{abstract}
Two hundred and nine samples were collected to study Coilia mystus. It is found that the maximum body length and weight are $182 \mathrm{~mm}$ and $23.4 \mathrm{~g}$ respectively in commercial stock. The minimum body length and weight $94 \mathrm{~mm}$ and $3.5 \mathrm{~g}$ respectively, and the individual average $K$ value $3.15 \times 10^{-4}$. The relationship between body length and body weight of Coilia mystus may be expressed as $W=1.174 \times 10^{-5} \mathrm{~L}^{2.773}$.

The individual average absolute fecundity and maturity coefficient of female are 7403. 48 eggs and $18.92 \%$ respectively. The individual average maturity coefficient of male is 8 . $02 \%$. The relationship between body length or body weight and absolute fecundity can be expressed as $F=1.906 \times 10^{-2} L^{2.54}$ and $F=688.13 W-2119.68$ respectivaly.
\end{abstract}

Key words Coilia mystus, propagating population, biological characteristics, relationship of factors 